\title{
Composition of Organic Compounds Adsorbed on PM10 in the Air Above Maribor
}

\author{
Alen Miuc, ${ }^{1, *}$ Ernest Vončina ${ }^{2}$ and Uroš Lešnik ${ }^{2}$ \\ ${ }^{1}$ Faculty of Chemistry and Chemical Engineering, University of Maribor, Smetanova 17, 2000 Maribor, Slovenia \\ ${ }^{2}$ National Laboratory of Health, Environment and Food, Prvomajska 1, 2000 Maribor, Slovenia \\ * Corresponding author: E-mail: alen.miuc@gmail.com
}

Received: 18-03-2015

\begin{abstract}
Organic compounds in atmospheric particulate matter above Maribor were analysed in 120 samples of PM10 sampled according to the EN 12341:2014 reference method. Organic compounds compositions were investigated together with the primary and secondary sources of air pollution. Silylation as derivatisation method was used for the GC-MS determination of volatile and semi-volatile polar organic compounds. Distribution of fatty acids, $n$-alkanes and iso-alkanes, phthalate esters, siloxanes, different sterols, various sugars and sugar alcohols, compounds of lignin and resin acids, dicarboxylic acids from photochemical reactions, PAHs, organic nitrogen compounds and products from secondary oxidation of monoterpenes were determined. The use of silicone grease for the purpose of lubricating the impact surface of the air sampler caused higher values of gravimetric determination. Solid particles may have been bounced from the surface of a greasy impact plate and re-entrained within the air stream and then collected on a sample filter. The carryover of siloxanes was at least from $5 \%$ up to $15 \%$ of the accumulated particles weight, depending on ambient temperature. This was the reason that the gravimetric results for determination of PM10 according to the standard EN 12341:2014 were overestimated.
\end{abstract}

Keywords: PM10, EN 12341:2014, secondary organic aerosol, volatile organic compounds

\section{Introduction}

Throughout the member States of Europe there is valid a uniform legislation on regulating the environmental area and the protection of human health. Member States are obliged to carry out measurements of air pollutants and keep the local public informed about the air quality. This data must be reported to the European Environmental Agency. In those cases where pollutants exceed the threshold values the state must design and implement measures to improve the conditions. ${ }^{1-4}$ Air Quality in Slovenia is determined by local emissions and the transport of polluted air across borders. ${ }^{3}$ In the air quality directive 2008/EC/50 the European Union has set two limit values for particulate matter with aerodynamic diameter of $10 \mu \mathrm{m}$ or less (PM10). ${ }^{1}$ The PM10 daily mean value may not exceed $50 \mu \mathrm{g} / \mathrm{m}^{3}$ more than 35 times during a year and the PM10 annual mean value may not exceed $40 \mu \mathrm{g} / \mathrm{m}^{3}$. Celje is the most polluted Slovenian town regarding the particulate matter PM10 with an average daily concentration of $45 \mu \mathrm{g} / \mathrm{m}^{3}$ and 41 exceedances of daily concentrations limits during the year 2014. In the year 2014 Maribor exceeded the daily concentrations limits 25 times with the PM10 annual mean value of 26.5 $\mu \mathrm{g} / \mathrm{m}^{3}{ }^{2}$ Air quality is usually the worst from November to March. The daily limits are exceeded mainly in winter due to increased traffic, domestic heating, industrial processes and meteorological phenomena such as inversion or low wind speed. ${ }^{1-5}$

Airborne suspended particulate matter can be of natural (forests, pollen, rainfall, storm, vegetation, volcanic ash...) or anthropogenic origin (emissions of industry, transport, burning of fossil fuels, biomass, agriculture...). Primary particles are released into the atmosphere from sources on the Earth's surface. ${ }^{6,7}$ A major fraction of organic aerosol load within the atmosphere is attributable to secondary organic aerosols (SOA) which are formed via gas phase oxidation of volatile organic compounds (VOCs) by atmospheric oxidants such as $\mathrm{O}_{3}, \mathrm{OH}$-radicals and $\mathrm{NO}_{3}$ radicals and by further gas-to-particle conversion processes. Photochemical processes can affect the chemical 
composition of organic compounds and their physicochemical properties such as volatility, hygroscopicity or the cloud condensation activities of SOA compounds. Three types of chemical processing can occur: fragmentation, functionalisation, and oligomerisation. Fragmentation will ultimately lead to degradation of a compound to a smaller, more volatile one. Functionalisation will basically introduce more oxygen containing groups and lead to the formation of less volatile, more polar oxygenated organic aerosol. During oligomerisation compounds with higher molecular mass are formed. ${ }^{7,8,9}$ The urban population is exposed to excessive concentrations of particulate matter, $\mathrm{CO}, \mathrm{NO}_{\mathrm{x}}, \mathrm{O}_{3}$ and different organic compounds. Even indoors within homes there are high concentrations of particulate matter due to internal sources (cooking, heating, smoking...) and the impact of external sources. Soot particles can carry toxic material such as polycyclic aromatic hydrocarbons (PAH) and heavy metals on their surfaces. ${ }^{9}$

The chemical composition of aerosols is a key factor for understanding the link between aerosols, clouds and climate. Carbonaceous aerosols are a subgroup of atmospheric aerosols, that consist of elemental carbon and organic compounds. ${ }^{10}$ Bio aerosols are particles that originate from vegetation. Aerosols affect human health, climate and visibility (smog, fog). Chemical compounds from industry and transport react in the atmosphere with ozone, $\mathrm{OH}, \mathrm{NO}_{3}$ and other radicals to form SOA. Together with the primary emissions they form the photochemical smog, which could be a risk to human health. ${ }^{11}$

Standard EN 12341:2014 is a reference method for sampling and measurements of the suspended fraction of PM10. This method is based on collecting the suspended fraction of particles with a diameter of $10 \mu \mathrm{m}$ or less (PM10) on a filter with a low volume sampler and gravimetric mass determination. ${ }^{12}$ The aim of our study was that after the gravimetric determination of PM10 also the chemical composition of organic compounds adsorbed on PM10 would be determined. This can provide us with better insight in the atmosphere and the possibility to identify harmful organic substances in the air above Maribor.

\section{Materials and Methods / Experimental Section}

Sample Collection and Preparation: The research included 120 samples of particulate matter PM10 collected according to standard EN 12341:2014 using low-volume air samplers (TCR Tecora Skypost PM, Leckel SEQ 47/50) with a flow rate of $38.3 \mathrm{~L} / \mathrm{min}$. Silicon-Hochvakuumfett (Merck 100g, CAS Nr. 107922) was used as a coating for the impact plate. 36 samples were obtained in winter, 34 in summer, 27 in spring and 23 in autumn. 30 samples were additionally obtained using polyphenylether
(Santovac 5, CAS Nr. 2455-71-2) as a coating for the impact plate. Aerosol samples were collected on quartz filters (Munktell, quartz microfiber discs, $47 \mathrm{~mm}$ ) during the period from July 2013 to August 2014 at eight locations in Maribor and its surroundings. The sampling sites are illustrated in Fig. 1. All experimental devices including a lowvolume air sampler probe and glassware were pre-extracted using dichloromethane. A reagent blank was analysed before sample analysis in each batch. Quartz filters were combusted before use at $500{ }^{\circ} \mathrm{C}$ for $6 \mathrm{~h}$. The benefit of using combusted quartz filters over the non-combusted filters is the reduced contamination.

After the gravimetric weighing of PM10 particles (Standard EN 12341:2014) the collected samples were placed in a glass vial with a Teflon cap and stored at $-20{ }^{\circ} \mathrm{C}$ prior to analysis. Samples were extracted three times $(3 \times$ $20 \mathrm{~mL}$ ) using a shaker with orbital movement having a mixture of dichloromethane/methanol $(2: 1 ; v / v)$. On a rotary evaporator the combined extracts were evaporated to dryness. Dry residues of extracts were dissolved in pyridine and derivatised with MSTFA ( $N$-methyl- $N$-trimethylsilyl trifluoroacetamide) for $1 \mathrm{~h}$ at $60{ }^{\circ} \mathrm{C}$. Hexatriacontane (nC36) was used as a compound of the external standard. Concentrated and derivatised extracts with a final volume of $100 \mu \mathrm{L}$ were quantitatively transferred into a glass vial and analysed using GC-MS.

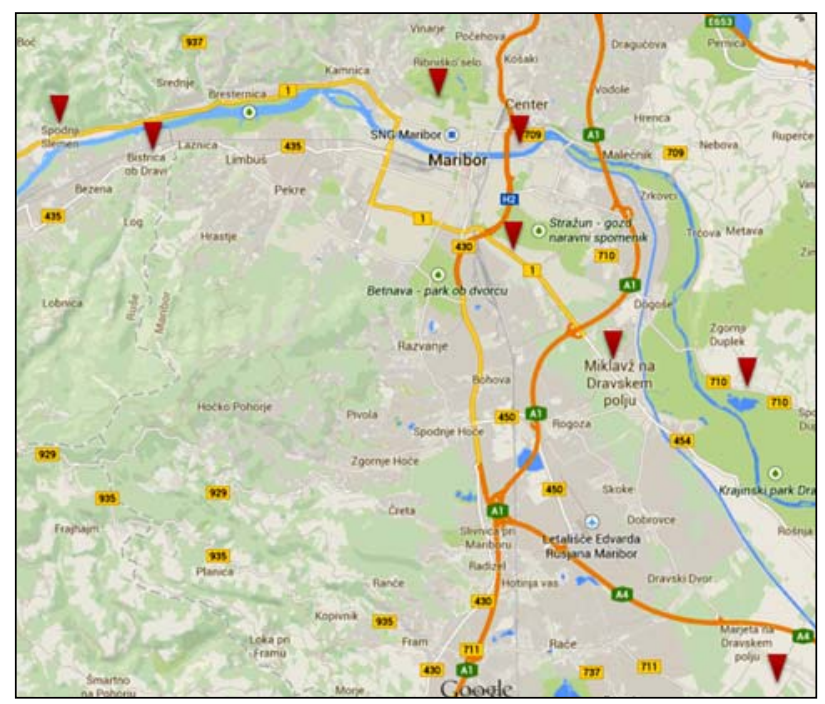

Figure 1. Sampling sites of PM10 collection in Maribor and its near surroundings (Sp. Slemen, Vrbanski plato, Bistrica ob Dravi, MB Center, MB NLZOH, Miklavž, Trniče, Duplek).

Instrumental Analysis: An Agilent (5973) mass spectrometer was used coupled to a gas chromatograph Agilent (6890) and Agilent autosampler (7683). For the chromatographic separation an Agilent DB-UI 8270 D capillary column was used $(30 \mathrm{~m} \times 250 \mu \mathrm{m}$ i.d., $0.25 \mu \mathrm{m}$ film thickness). 
The temperature program was the following: 0.75 min at a temperature of $105^{\circ} \mathrm{C}, 30^{\circ} \mathrm{C} / \mathrm{min}$ up to $120^{\circ} \mathrm{C}$ $(0.1 \mathrm{~min}), 2.7^{\circ} \mathrm{C} / \mathrm{min}$ to $320^{\circ} \mathrm{C}(5 \mathrm{~min})$. The carrier gas was helium (He 6.0, Messer Austria) at a constant flow of $0.9 \mathrm{ml} / \mathrm{min}$. The ion source temperature was $250^{\circ} \mathrm{C}$. The injection port and transfer line were kept at $290^{\circ} \mathrm{C}$. The mass spectrometer was operated in electron ionisation (EI) mode at $70 \mathrm{eV}$ and scanned in full scan mode in the range 70-800 Da. Chromatograms were processed by a computer program AMDIS (Automated Mass Spectral Deconvolution And Identification System Software). Detected compounds were identified by comparing their spectra with those, reported in the Willey and NIST (W9N08) mass spectra libraries or with data from literature.

\section{Results and Discussion}

In the sample extracts the following compounds were detected: fatty acids, sugars, sugar alcohols and higher alkanols, compounds of lignin and wood resins, dicarboxylic acids, distribution of $n$-alkanes and iso-alkanes, phthalate esters, siloxanes, sterols, polycyclic aromatic hydrocarbons, urea as an organic nitrogen compound and products from secondary oxidation of monoterpene compounds.

Emissions from individual sources depended on the season. We detected indicators (tracer compounds) for combustion of biomass (levoglucosan), lignin and wood resins (dehydro-abietic acid and vanillic acid), secondary biogenic aerosols (pinonic acid) and fecal pollution from livestock and aeration of biological treatment plants (cholesterol). From the review of the detected compounds it could be seen that the most abundant compounds during the summer season were the derivatives of sugars, sugar alcohols and free fatty acids. The comparison between the chromatograms of all the samples' extracts showed that the composition of aerosols was very similar in warmer seasons with a predominance of biogenic compounds over anthropogenic compounds. During winter the anthropogenic organic compounds dominated on PM10 sample extracts, the source of which was the combustion of fossil fuels for heating or transport.

\section{1. Free Fatty Acids}

A set of silylated derivatives of free fatty acids (FFA) from pentanoic acid up to hexacosanoic acid is shown in Figure 3. FFA had characteristic mass fragments

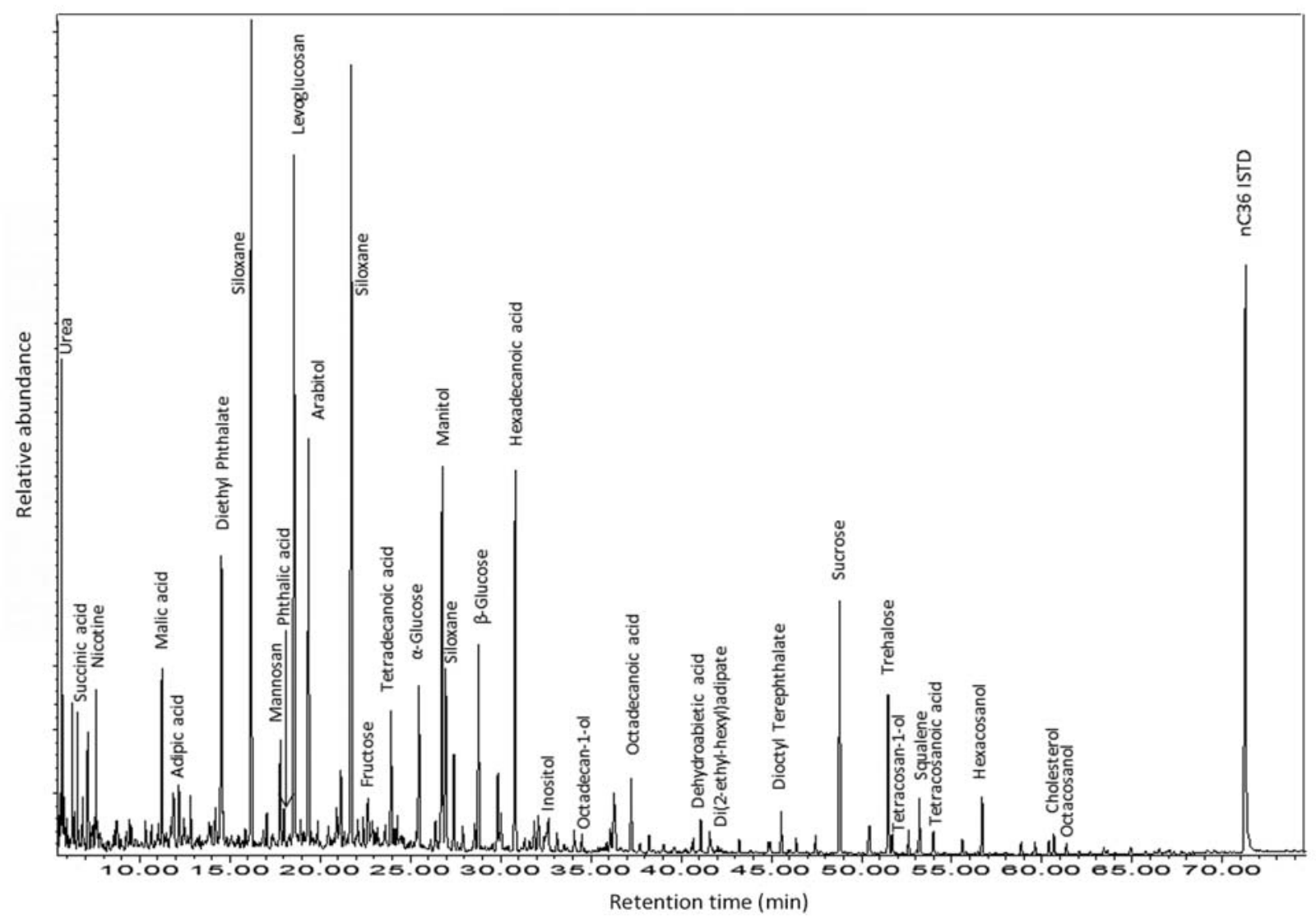

Figure 2. Typical GC/MS chromatogram of silylated PM10 sample extract from Maribor (Collected: 18.7.2014, MB NLZOH). 
Table 1. Compounds detected in concentrated silylated sample extracts in the air above Maribor.

\begin{tabular}{|c|c|c|c|c|c|}
\hline & Compound & Formula & M.W. & Major ions (m/z) & Source/tracer/ industrial use \\
\hline $\begin{array}{l}n \text {-Alkanoic } \\
\text { acids }\end{array}$ & $\begin{array}{l}\text { Pentanoic acid } \\
\text { Hexanoic acid } \\
\text { Heptanoic acid } \\
\text { Octanoic acid } \\
\text { Nonanoic acid } \\
\text { Decanoic acid } \\
\text { Undecanoic acid } \\
\text { Dodecanoic acid } \\
\text { Tridecanoic acid } \\
\text { Tetradecanoic acid } \\
\text { Pentadecanoic acid } \\
\text { Hexadecanoic acid } \\
\text { Heptadecanoic acid } \\
\text { Octadecanoic acid } \\
\text { Eicosanoic acid } \\
\text { Tetracosanoic acid } \\
\text { Hexacosanoic acid }\end{array}$ & $\begin{array}{l}\mathrm{C}_{5} \mathrm{H}_{10} \mathrm{O}_{2} \\
\mathrm{C}_{6} \mathrm{H}_{12} \mathrm{O}_{2} \\
\mathrm{C}_{7} \mathrm{H}_{14} \mathrm{O}_{2} \\
\mathrm{C}_{8} \mathrm{H}_{16} \mathrm{O}_{2} \\
\mathrm{C}_{9} \mathrm{H}_{18} \mathrm{O}_{2} \\
\mathrm{C}_{10} \mathrm{H}_{20} \mathrm{O}_{2} \\
\mathrm{C}_{11} \mathrm{H}_{22} \mathrm{O}_{2} \\
\mathrm{C}_{12} \mathrm{H}_{24} \mathrm{O}_{2} \\
\mathrm{C}_{13} \mathrm{H}_{26} \mathrm{O}_{2} \\
\mathrm{C}_{14} \mathrm{H}_{28} \mathrm{O}_{2} \\
\mathrm{C}_{15} \mathrm{H}_{30} \mathrm{O}_{2} \\
\mathrm{C}_{16} \mathrm{H}_{32} \mathrm{O}_{2} \\
\mathrm{C}_{17} \mathrm{H}_{34} \mathrm{O}_{2} \\
\mathrm{C}_{18} \mathrm{H}_{36} \mathrm{O}_{2} \\
\mathrm{C}_{20} \mathrm{H}_{40} \mathrm{O}_{2} \\
\mathrm{C}_{24} \mathrm{H}_{48} \mathrm{O}_{2} \\
\mathrm{C}_{26} \mathrm{H}_{52} \mathrm{O}_{2}\end{array}$ & $\begin{array}{l}102 \\
116 \\
130 \\
146 \\
166 \\
176 \\
190 \\
200 \\
214 \\
228 \\
242 \\
256 \\
270 \\
284 \\
312 \\
368 \\
396\end{array}$ & $\begin{array}{l}117,159 \\
117,173 \\
117,187 \\
117,201 \\
117,215 \\
117,229 \\
117,243 \\
117,257 \\
117,271 \\
117,285 \\
117,299 \\
117,313 \\
117,327 \\
117,341 \\
117,369 \\
117,425 \\
117,453\end{array}$ & $\begin{array}{l}<\mathrm{C} 20 \text { : } 0 \text { Burning of fossil } \\
\text { fuels, microbial activity } \\
\text { and cooking }\end{array}$ \\
\hline $\begin{array}{l}n \text {-Alkenoic } \\
\text { acids }\end{array}$ & $\begin{array}{l}\text { Hexadec-9-enoic acid } \\
\text { 9,12-Octadecadienoic acid } \\
\text { Octadec-9-enoic acid }\end{array}$ & $\begin{array}{l}\mathrm{C}_{16} \mathrm{H}_{30} \mathrm{O}_{2} \\
\mathrm{C}_{18} \mathrm{H}_{32} \mathrm{O}_{2} \\
\mathrm{C}_{18} \mathrm{H}_{34} \mathrm{O}_{2}\end{array}$ & $\begin{array}{l}254 \\
280 \\
282\end{array}$ & $\begin{array}{l}117,311 \\
117,337 \\
117,339\end{array}$ & \\
\hline $\begin{array}{l}\text { Dicarboxylic } \\
\text { acids }\end{array}$ & $\begin{array}{l}\text { Succinic acid } \\
\text { Malic acid } \\
\text { Glutaric acid } \\
\text { Adipic acid } \\
\text { Phthalic acid } \\
\text { Azelaic acid } \\
\text { Citric acid } \\
\text { Quinic acid } \\
\text { Di(2-ethyl-hexyl)adipate }\end{array}$ & $\begin{array}{l}\mathrm{C}_{4} \mathrm{H}_{6} \mathrm{O}_{4} \\
\mathrm{C}_{4} \mathrm{H}_{6} \mathrm{O}_{5} \\
\mathrm{C}_{5} \mathrm{H}_{8} \mathrm{O}_{4} \\
\mathrm{C}_{6} \mathrm{H}_{10} \mathrm{O}_{4} \\
\mathrm{C}_{8} \mathrm{H}_{6} \mathrm{O}_{4} \\
\mathrm{C}_{9} \mathrm{H}_{16} \mathrm{O}_{4} \\
\mathrm{C}_{6} \mathrm{H}_{8} \mathrm{O}_{7} \\
\mathrm{C}_{7} \mathrm{H}_{12} \mathrm{O}_{6} \\
\mathrm{C}_{22} \mathrm{H}_{42} \mathrm{O}_{4}\end{array}$ & $\begin{array}{l}118 \\
134 \\
132 \\
146 \\
166 \\
188 \\
192 \\
192 \\
370\end{array}$ & $\begin{array}{l}147,247 \\
147,233 \\
147,349 \\
147,275 \\
147,295 \\
147,317 \\
147,273 \\
147,345 \\
147,112\end{array}$ & $\begin{array}{l}\text { Aliphatic olefins } \\
\text { Aliphatic olefins } \\
\text { Carboxylic acids } \\
\text { Polyol }\end{array}$ \\
\hline n-Alkanols & $\begin{array}{l}\text { Hexadecanol } \\
\text { Octadecanol } \\
\text { Docosanol } \\
\text { Tetracosanol } \\
\text { Hexacosanol } \\
\text { Octacosanol }\end{array}$ & $\begin{array}{l}\mathrm{C}_{16} \mathrm{H}_{34} \mathrm{O} \\
\mathrm{C}_{18} \mathrm{H}_{38} \mathrm{O} \\
\mathrm{C}_{22} \mathrm{H}_{46} \mathrm{O} \\
\mathrm{C}_{24} \mathrm{H}_{50} \mathrm{O} \\
\mathrm{C}_{26} \mathrm{H}_{54} \mathrm{O} \\
\mathrm{C}_{28} \mathrm{H}_{58} \mathrm{O}\end{array}$ & $\begin{array}{l}242 \\
270 \\
326 \\
354 \\
382 \\
410\end{array}$ & $\begin{array}{l}103,299 \\
103,327 \\
103,383 \\
103,411 \\
103,439 \\
103,467\end{array}$ & $\begin{array}{l}\text { Wax coatings of higher } \\
\text { plants, deciduous and } \\
\text { coniferous trees, biomass }\end{array}$ \\
\hline$n$-Alkanes & $\begin{array}{l}\text { Dodecane } \\
\text { Tridecane } \\
\text { Tetradecane } \\
\text { Pentadecane } \\
\text { Hexadecane } \\
\text { Heptadecane } \\
\text { Octadecane } \\
\text { Nonadecane } \\
\text { Eicosane } \\
\text { Heneicosane } \\
\text { Docosane } \\
\text { Tricosane } \\
\text { Tetracosane } \\
\text { Pentacosane } \\
\text { Hexacosane } \\
\text { Heptacosane } \\
\text { Octacosane } \\
\text { Nonacosane } \\
\text { Triacontane } \\
\text { Hentriacontane }\end{array}$ & $\begin{array}{l}\mathrm{C}_{12} \mathrm{H}_{26} \\
\mathrm{C}_{13} \mathrm{H}_{28} \\
\mathrm{C}_{14} \mathrm{H}_{30} \\
\mathrm{C}_{15} \mathrm{H}_{32} \\
\mathrm{C}_{16} \mathrm{H}_{34} \\
\mathrm{C}_{17} \mathrm{H}_{36} \\
\mathrm{C}_{18} \mathrm{H}_{38} \\
\mathrm{C}_{19} \mathrm{H}_{40} \\
\mathrm{C}_{20} \mathrm{H}_{42} \\
\mathrm{C}_{21} \mathrm{H}_{44} \\
\mathrm{C}_{22} \mathrm{H}_{46} \\
\mathrm{C}_{23} \mathrm{H}_{48} \\
\mathrm{C}_{24} \mathrm{H}_{50} \\
\mathrm{C}_{25} \mathrm{H}_{52} \\
\mathrm{C}_{26} \mathrm{H}_{54} \\
\mathrm{C}_{27} \mathrm{H}_{56} \\
\mathrm{C}_{28} \mathrm{H}_{58} \\
\mathrm{C}_{29} \mathrm{H}_{60} \\
\mathrm{C}_{30} \mathrm{H}_{62} \\
\mathrm{C}_{31} \mathrm{H}_{64}\end{array}$ & $\begin{array}{l}170 \\
184 \\
198 \\
212 \\
226 \\
240 \\
254 \\
268 \\
282 \\
296 \\
310 \\
324 \\
338 \\
352 \\
366 \\
380 \\
394 \\
408 \\
422 \\
436\end{array}$ & $\begin{array}{l}85,170 \\
85,184 \\
85,198 \\
85,212 \\
85,226 \\
85,240 \\
85,254 \\
85,268 \\
85,282 \\
85,296 \\
85,310 \\
85,324 \\
85,338 \\
85,352 \\
85,366 \\
85,380 \\
85,394 \\
85,408 \\
85,422 \\
85,436\end{array}$ & $\begin{array}{l}\mathrm{C}_{15}-\mathrm{C}_{37} \text { (odd }>\text { even) microbial, } \\
\text { plant waxes } \\
\mathrm{C}_{16}-\mathrm{C}_{40+} \\
\text { (odd<even) plastics }\end{array}$ \\
\hline
\end{tabular}




\begin{tabular}{|c|c|c|c|c|c|}
\hline & Compound & Formula & M.W. & Major ions (m/z) & Source/tracer/ industrial use \\
\hline & Dotriacontane & $\mathrm{C}_{32} \mathrm{H}_{66}$ & 450 & 85,450 & \\
\hline & Tritriacontane & $\mathrm{C}_{33} \mathrm{H}_{68}$ & 464 & 85,464 & \\
\hline & Tetratriacontane & $\mathrm{C}_{34} \mathrm{H}_{70}$ & 478 & 85,478 & \\
\hline & Pentatriacontane & $\mathrm{C}_{35} \mathrm{H}_{72}$ & 492 & 85,492 & \\
\hline \multirow{12}{*}{$\begin{array}{l}\text { Sugars and } \\
\text { sugar alcohols }\end{array}$} & Galactosan & $\mathrm{C}_{6} \mathrm{H}_{10} \mathrm{O}_{5}$ & 162 & 204,217 & Cellulose and \\
\hline & Mannosan & $\mathrm{C}_{6} \mathrm{H}_{10} \mathrm{O}_{5}$ & 162 & 204,217 & Hemicellulose \\
\hline & Levoglucosan & $\mathrm{C}_{6} \mathrm{H}_{10} \mathrm{O}_{5}$ & 162 & 204,333 & Biomass burning \\
\hline & Arabitol & $\mathrm{C}_{5} \mathrm{H}_{12} \mathrm{O}_{5}$ & 152 & 204,217 & Fungal spores \\
\hline & 1,6-anhydro Glukopyranose & $\mathrm{C}_{6} \mathrm{H}_{10} \mathrm{O}_{5}$ & 162 & 204,217 & \multirow{8}{*}{$\begin{array}{l}\text { Soil resuspension } \\
\text { and microbiota } \\
\text { Fungal spores }\end{array}$} \\
\hline & Fructose & $\mathrm{C}_{6} \mathrm{H}_{12} \mathrm{O}_{6}$ & 180 & 204,217 & \\
\hline & $\alpha$-Glucose & $\mathrm{C}_{6} \mathrm{H}_{12} \mathrm{O}_{6}$ & 180 & 204,217 & \\
\hline & Mannitol & $\mathrm{C}_{6} \mathrm{H}_{14} \mathrm{O}_{6}$ & 182 & 204,217 & \\
\hline & $\beta$-Glucose & $\mathrm{C}_{6} \mathrm{H}_{12} \mathrm{O}_{6}$ & 180 & 204,217 & \\
\hline & Inositol & $\mathrm{C}_{6} \mathrm{H}_{12} \mathrm{O}_{6}$ & 180 & 204,217 & \\
\hline & Sucrose & $\mathrm{C}_{12} \mathrm{H}_{22} \mathrm{O}_{11}$ & 342 & 204,361 & \\
\hline & Trehalose & $\mathrm{C}_{12} \mathrm{H}_{22} \mathrm{O}_{11}$ & 342 & 204,361 & \\
\hline Lignin and & 4-Hydoxybenzoic acid & $\mathrm{C}_{7} \mathrm{H}_{6} \mathrm{O}_{3}$ & 138 & 267,282 & Lignin \\
\hline Resin & Vanillin & $\mathrm{C}_{8} \mathrm{H}_{8} \mathrm{O}_{3}$ & 152 & 194,209 & Conifers \\
\hline \multirow[t]{4}{*}{ products } & Vanillic acid & $\mathrm{C}_{8} \mathrm{H}_{8} \mathrm{O}_{4}$ & 168 & 297,312 & Conifers \\
\hline & Syringaldehyde & $\mathrm{C}_{9} \mathrm{H}_{10} \mathrm{O}_{4}$ & 182 & 224,254 & Hardwood \\
\hline & Syringic acid & $\mathrm{C}_{9} \mathrm{H}_{10} \mathrm{O}_{5}$ & 198 & 297,327 & Hardwood \\
\hline & Dehydroabietic acid & $\mathrm{C}_{20} \mathrm{H}_{28} \mathrm{O}_{2}$ & 300 & 239,357 & Conifers \\
\hline \multirow[t]{5}{*}{ Phthalates } & Diethyl Phthalate & $\mathrm{C}_{12} \mathrm{H}_{14} \mathrm{O}_{4}$ & 222 & 149,177 & Plasticizer, aerosol sprays \\
\hline & Dibutyl Phthalate & $\mathrm{C}_{16} \mathrm{H}_{22} \mathrm{O}_{4}$ & 278 & 149,117 & Cosmetics \\
\hline & Benzylbutyl Phthalate & $\mathrm{C}_{19} \mathrm{H}_{20} \mathrm{O}_{4}$ & 312 & 149 & Plasticizer \\
\hline & Diethylhexyl phthalate & $\mathrm{C}_{24} \mathrm{H}_{38} \mathrm{O}_{4}$ & 390 & 149,279 & Plasticizer \\
\hline & $\begin{array}{l}\left(\mathrm{C}_{10}-\mathrm{C}_{21}\right) \text { Alkylsulfonic } \\
\text { phenyl (ester) }\end{array}$ & & & 94,293 & $\begin{array}{l}\text { Replacement of } \\
\text { bis(2-ethylhexyl) phthalate }\end{array}$ \\
\hline SOA & Sabina ketone & $\mathrm{C}_{9} \mathrm{H}_{14} \mathrm{O}$ & 138 & 81 & Sabinene \\
\hline products & Nopinone & $\mathrm{C}_{9} \mathrm{H}_{14} \mathrm{O}$ & 138 & 83 & $\beta$-pinene \\
\hline \multirow[t]{3}{*}{ from terpenes } & p-Cymene & $\mathrm{C}_{10} \mathrm{H}_{14}$ & 134 & 119 & Monoterpenes \\
\hline & Pinonaldehyde & $\mathrm{C}_{10} \mathrm{H}_{16} \mathrm{O}_{2}$ & 168 & 83 & $\alpha$-Pinene \\
\hline & Pinonic acid & $\mathrm{C}_{10} \mathrm{H}_{16} \mathrm{O}_{3}$ & 184 & 83 & $\alpha$-Pinene \\
\hline \multirow[t]{12}{*}{ PAH } & Pyrene & $\mathrm{C}_{16} \mathrm{H}_{10}$ & 202 & 202,101 & \multirow{12}{*}{$\begin{array}{l}\text { Fossil fuels, incomplete } \\
\text { combustion of carbon } \\
\text { based fuels }\end{array}$} \\
\hline & Benzo[ghi]fluoranthene & $\mathrm{C}_{18} \mathrm{H}_{10}$ & 226 & 226,113 & \\
\hline & Benzo[a]anthracene & $\mathrm{C}_{18} \mathrm{H}_{12}$ & 228 & 228,114 & \\
\hline & Chrysene & $\mathrm{C}_{18} \mathrm{H}_{12}$ & 228 & 228,114 & \\
\hline & 7H-Benz[de]anthracen-7-one & $\mathrm{C}_{17} \mathrm{H}_{10} \mathrm{O}$ & 230 & 230,115 & \\
\hline & Benzo[k]fluoranthene & $\mathrm{C}_{20} \mathrm{H}_{12}$ & 252 & 252,126 & \\
\hline & Benzo[e]pyrene & $\mathrm{C}_{20} \mathrm{H}_{12}$ & 252 & 252,126 & \\
\hline & Benzo[a]pyrene & $\mathrm{C}_{20} \mathrm{H}_{12}$ & 252 & 252,126 & \\
\hline & Indeno[1,2,3-cd]pyrene & $\mathrm{C}_{22} \mathrm{H}_{12}$ & 276 & 276,138 & \\
\hline & Benzo[ghi]perylene & $\mathrm{C}_{22} \mathrm{H}_{12}$ & 276 & 276,138 & \\
\hline & Indeno [1,2,3-cd]fluoranthene & $\mathrm{C}_{22} \mathrm{H}_{12}$ & 276 & 276,138 & \\
\hline & Coronene & $\mathrm{C}_{24} \mathrm{H}_{12}$ & 300 & 300,150 & \\
\hline Other & Urea & $\mathrm{CH}_{4} \mathrm{~N}_{2} \mathrm{O}$ & 60 & 189,147 & Agricultural activities, SOA \\
\hline \multirow[t]{10}{*}{ compounds } & 2-methylthreitol & $\mathrm{C}_{5} \mathrm{H}_{12} \mathrm{O}_{4}$ & 136 & 219,321 & Isoprene \\
\hline & 2-methylerythritol & $\mathrm{C}_{5} \mathrm{H}_{12} \mathrm{O}_{4}$ & 136 & 219,321 & Isoprene \\
\hline & Nonanal & $\mathrm{C}_{9} \mathrm{H}_{18} \mathrm{O}$ & 142 & 98 & Biogenic SOA \\
\hline & Decanal & $\mathrm{C}_{10} \mathrm{H}_{20} \mathrm{O}$ & 156 & 112 & Biogenic SOA \\
\hline & Nicotine & $\mathrm{C}_{10} \mathrm{H}_{14} \mathrm{~N}_{2}$ & 162 & 84,161 & Smoking \\
\hline & Tricarballylic acid & $\mathrm{C}_{6} \mathrm{H}_{8} \mathrm{O}_{6}$ & 176 & 217,377 & SOA \\
\hline & 6,10,14-trimethyl-2-pentadecanone & $\mathrm{C}_{18} \mathrm{H}_{36} \mathrm{O}$ & 268 & 109,250 & Phytol \\
\hline & Cholesterol & $\mathrm{C}_{27} \mathrm{H}_{46} \mathrm{O}$ & 386 & 329,353 & Fecal pollution \\
\hline & Squalene & $\mathrm{C}_{30} \mathrm{H}_{50}$ & 410 & 129 & \\
\hline & $\beta$-Sitosterol & $\mathrm{C}_{29} \mathrm{H}_{50} \mathrm{O}$ & 414 & 357,486 & Vegetation in general \\
\hline
\end{tabular}




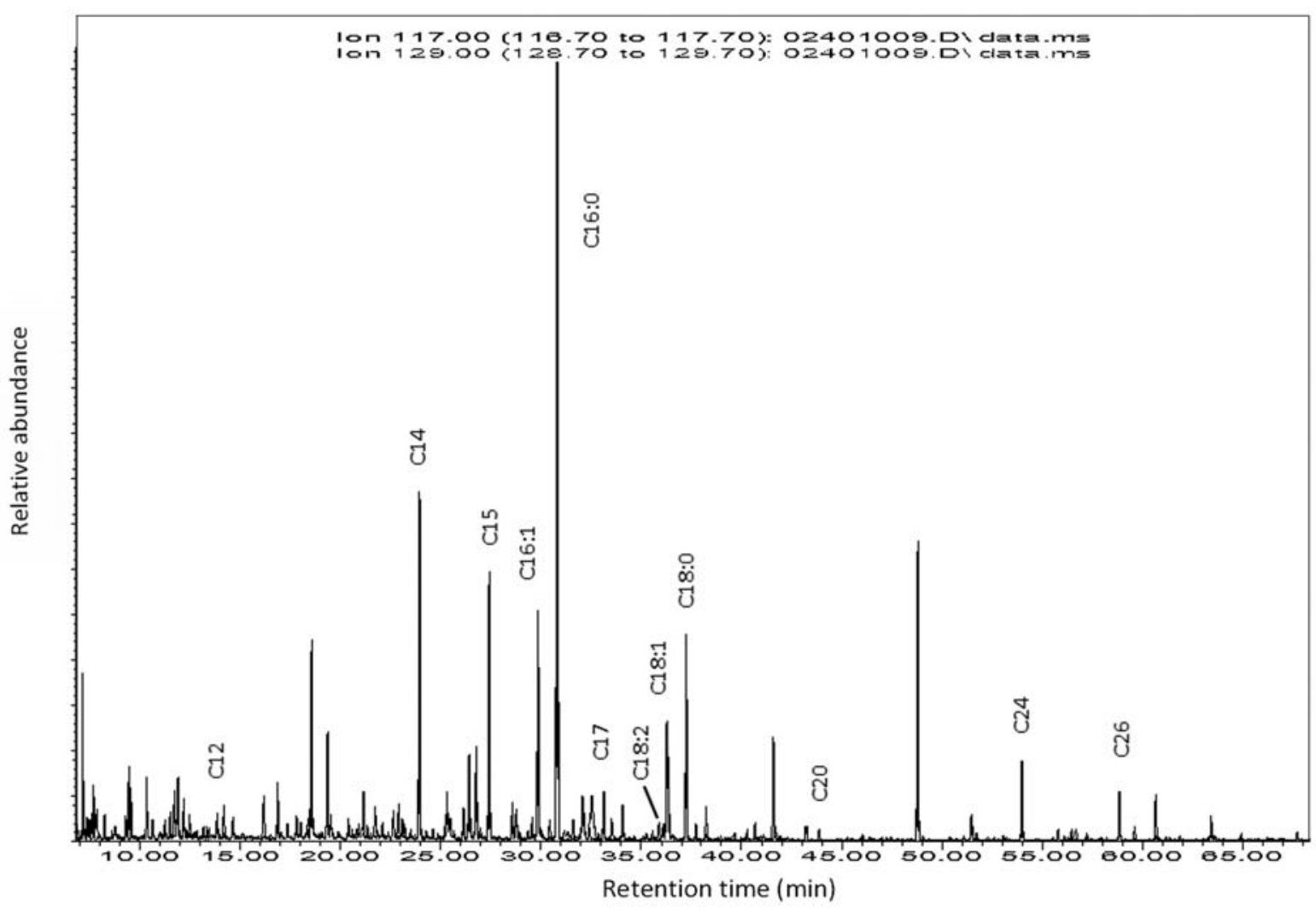

Figure 3. Chromatogram of free fatty acids distribution from sample extracts (Collected: 10.5.2014, MB VP).

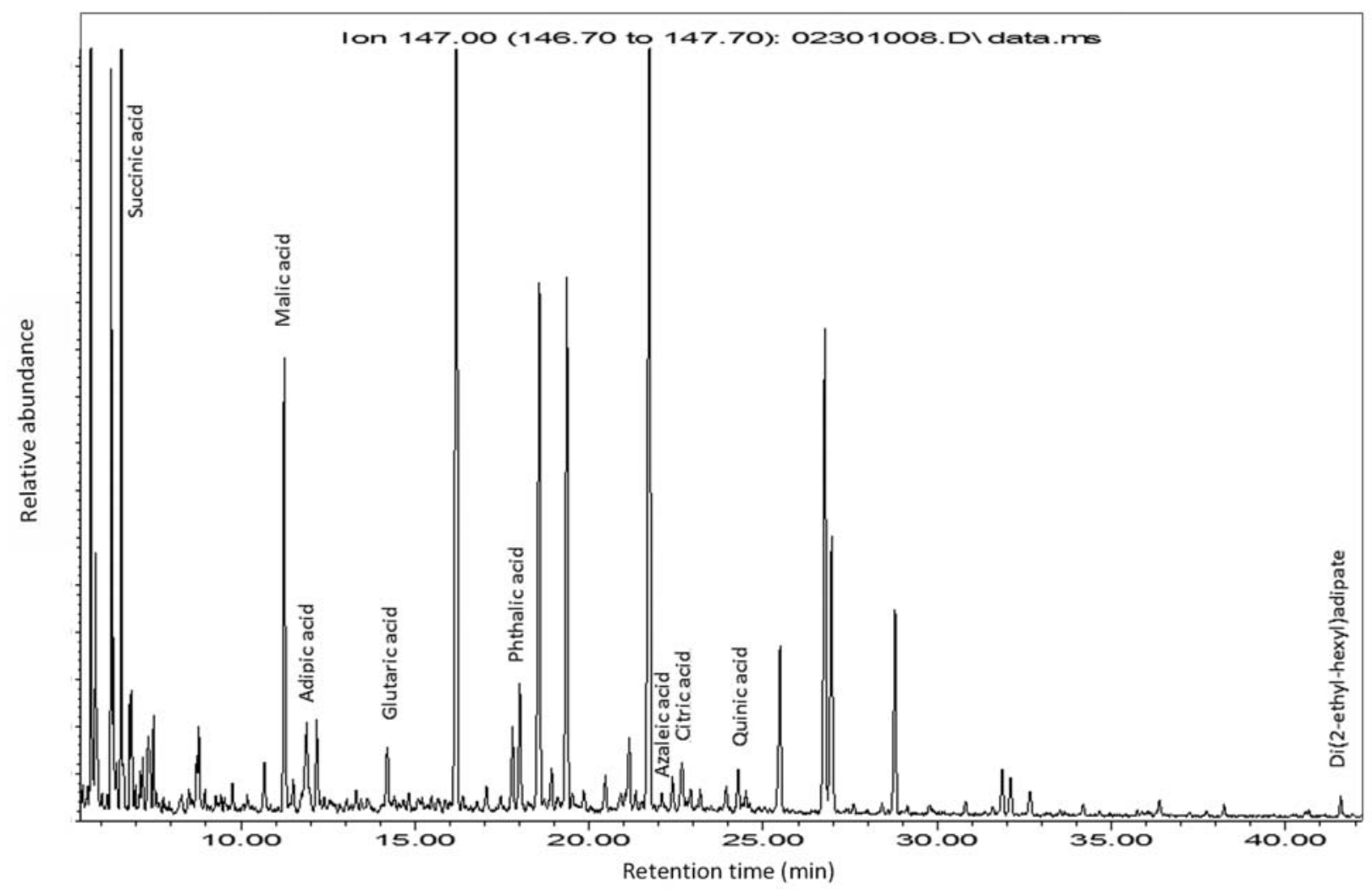

Figure 4. Typical chromatogram of dicarboxylic acids distribution from sample extracts (Collected: 10.5.2014, MB VP). 
$\mathrm{m} / \mathrm{z} 117$ and $\mathrm{m} / \mathrm{z}, 129$. Odd-numbered FFA were naturally present in trace amounts. Their presence in the sample extracts PM10 was attributed to decomposition of higher fatty acids and their photochemical transformation. ${ }^{13}$ The presence of fatty acids with low molecular weight $(<\mathrm{C} 20: 0)$ indicated burning of fossil fuels, microbial activity, and cooking. High molecular weight fatty acids are produced by the incineration of biomass which contains plant waxes. ${ }^{13,14}$ As anticipated palmitic (16:0) acid was the most abundant.

\section{2. Dicarboxylic Acids}

Emissions from motorised vehicles, combustion of biomass and oceanic emissions are the primary sources of dicarboxylic acids. One of the main sources of dicarboxylic acids are also atmospheric photochemical reactive processes. ${ }^{6,9,16}$ Lower dicarboxylic acids have different origins than higher dicarboxylic acids. ${ }^{10}$ Phthalic acid is an aromatic dicarboxylic acid that is released into the environment by biomass burning, vehicle emissions or as a by-product from the photochemical oxidation of polycyclic aromatic hydrocarbons and phthalate esters. The presence of glutaric and adipic acid indicate oxidative degradation of cyclic and aliphatic olefins, and azelaic acid on the presence of unsaturated carboxylic acids. ${ }^{6,13,15}$ Amongst anthropogenic compounds di(2-ethyl-hexyl)adipate and quinic acid as a polyol from cyclohexane and cyclohexanecarboxylic acid were also detected. ${ }^{6,16}$

\section{3. Alkanols}

All alkanols are presented with the fragment $\mathrm{m} / \mathrm{z} 75$ and $m / z$ 103. They range from hexadecanol up to octacosanol. Odd-alkanols were not detected. We associated the presence of alkanols with wax coatings of higher plants, deciduous and coniferous trees. ${ }^{17}$

\section{4. n-Alkanes}

Anthropogenic and biogenic primary sources such as vegetation waxes, incineration of biomass, cooking and exhaust gases of motor vehicles were sources of $n$-alkanes and iso-alkanes which can be detected on collected PM10 samples from dodecane (nC12) to pentatriacontane (nC35).$^{18}$ The carbon preference index (CPI) is a qualitative tool to assess the influences of biogenic and anthropogenic inputs for $n$-alkanes. The equation for CPI is a sum of the odd carbon number homologs (over a range), divided by the sum of even carbon homologs (over the same range). An odd-to-even carbon number predominance was observed for biologically sources of $n$-alkanes. A CPI $>3$ indicated a major recent release of biological components into the air. CPI values close to unity were observed regarding the presence of anthropogenic compounds. Petroleum-derived $n$-alkanes had CPI $\approx 1 .{ }^{16,17}$

The predominance of odd numbered $n$-alkanes during warmer seasons points to increased biological activity. This biological impact could be linked to the nearby Pohorje Mountain. $n$-alkanes of higher plants (nC25 to

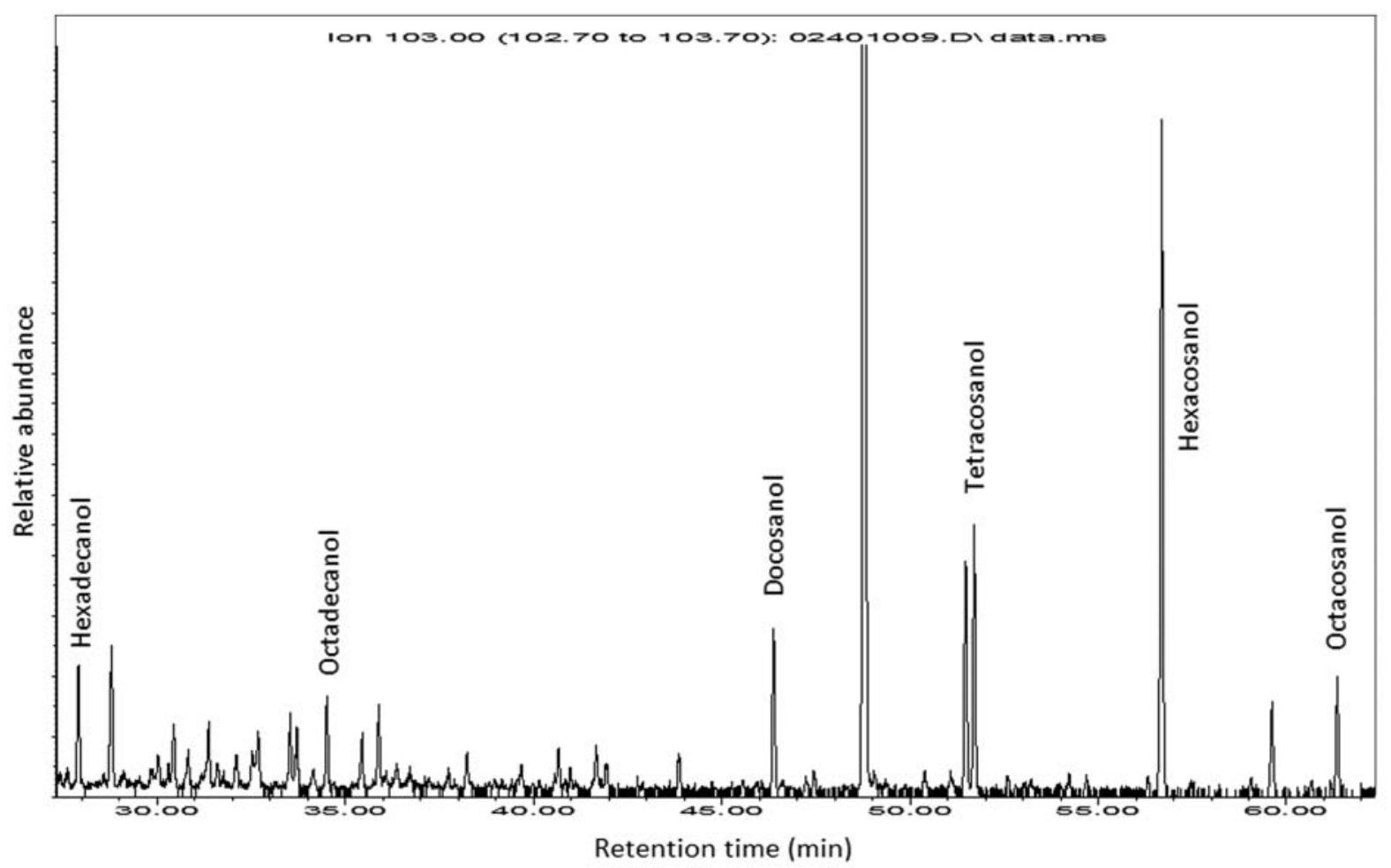

Figure 5. Chromatogram of fatty alcohols (alkanols) distribution from sample extracts (Collected: 19.7. 2014, NLZOH). 


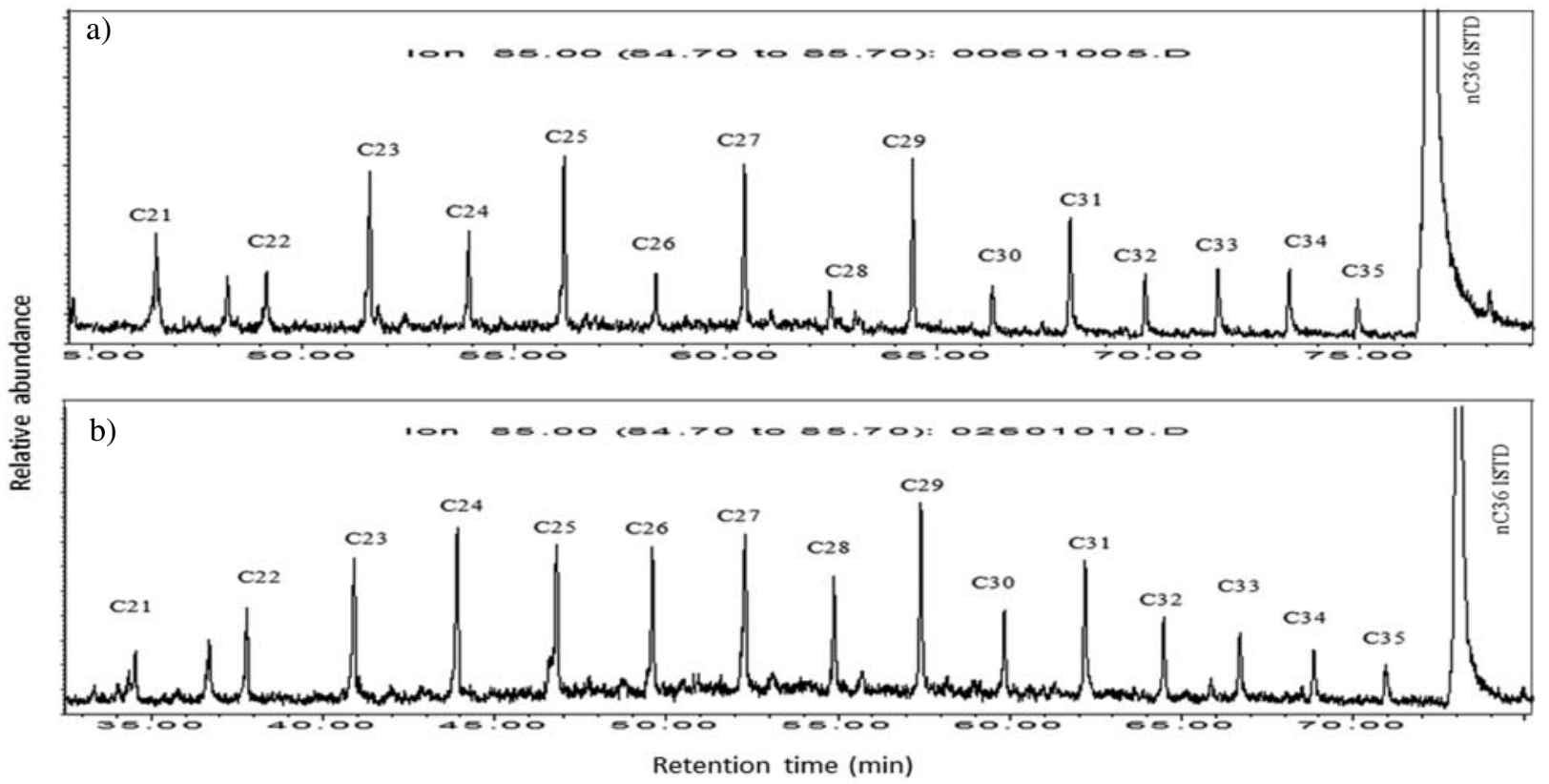

Figure 6. Typical chromatogram for distribution of n-alkanes from sample extracts collected on A) 18.7.2014 at MB NLZOH and B) 19.11.2013 at MB NLZOH.

nC35) are part of epicuticular wax layers and the waxes of needles from conifers. ${ }^{19,28} n$-alkanes with a lower molecular weight are characteristic for the car exhaust gases. The predominance of even $n$-alkanes is expected by pyrolytic decomposition of fossil fuels and burning. During the winter season we detected an increase of even numbered $n$-alkanes and a CPI close to 1 as the result of increased use of fossil fuels during the colder seasons.

\section{5. Sugars and Sugar Alcohols}

All sugars and sugar alcohols are presented with fragments $m / z 204$ and $m / z 217$, sucrose and glucose have a characteristic fragment $\mathrm{m} / \mathrm{z}, 361$. Biomass burning is a major source of atmospheric gases and particles on regional and global scales. Particles produced by biomass burning can influence the global climate. ${ }^{6}$ Levo-

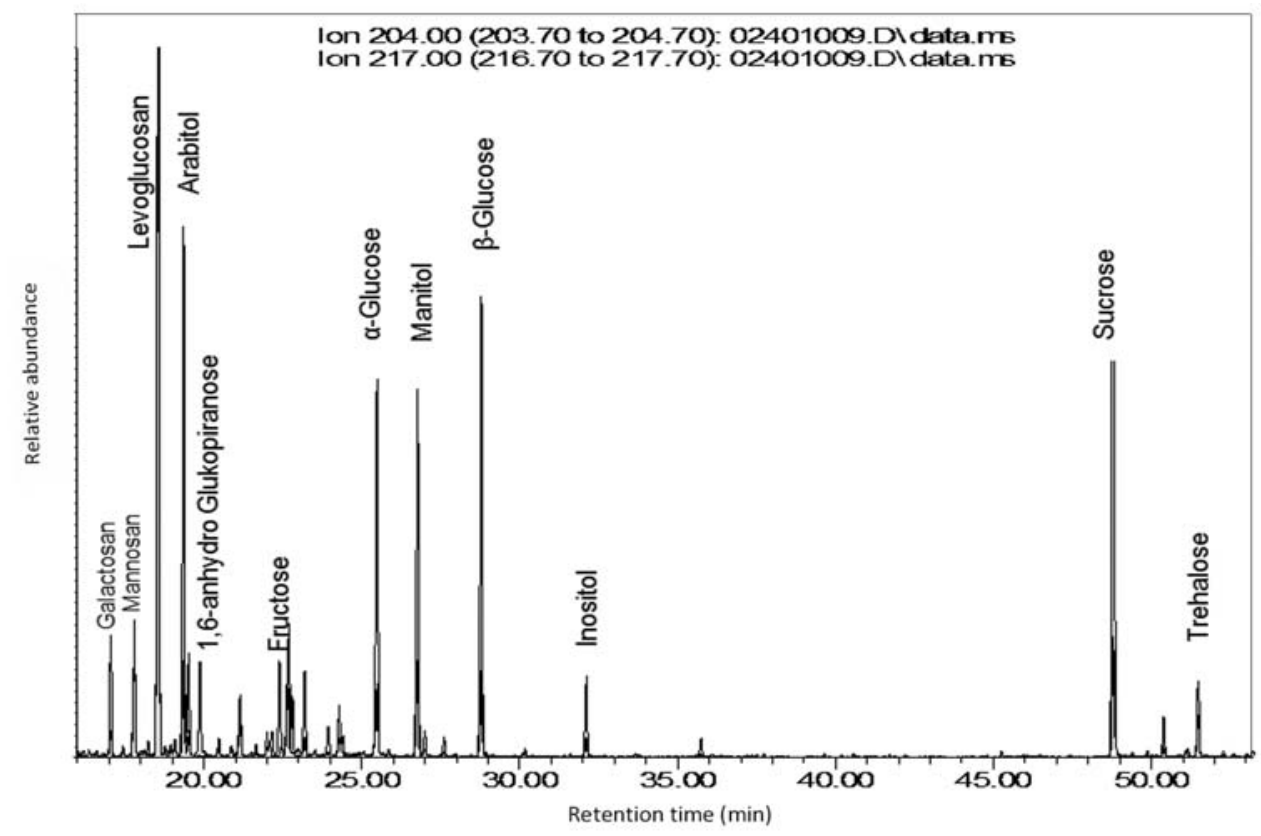

Figure 7. Total Ion current of mass fragments $\mathrm{m} / \mathrm{z}, 204$ and $\mathrm{m} / \mathrm{z} 217$ characteristic for sugars and sugar alcohols silylated derivatives from sample extracts (Collected: 12.5. 2014, MB VP). 
glucosan is produced by combustion of cellulose and is the key tracer compound for the incineration of biomass. Mannosan and galactosan are thermal breakdown products of cellulose and hemicellulose and were detected in all samples. ${ }^{13,16}$ Elevated concentrations of sugars in spring and summer were observed, suggesting enhanced biological activity during warmer seasons. In all PM10 samples glucose and sucrose are the dominant sugars. Arabitol and mannitol are proposed tracer compounds of fungal spores. Plant elements such as pollen, fungal spores, fruits and their fragments are a source of fructose, glucose and sucrose. Trehalose is present in micro-organisms (fungi, bacteria and yeast), invertebrates and in some plants. ${ }^{6,20}$ Trehalose is the most common disaccharide of earth soil and is a proposed tracer compound for re-suspension of soil particles and macadam roads. ${ }^{13}$

\section{6. Lignin and Resin Products}

Lignin is a major biopolymer of wood and upon burning yields aromatic phenols, aldehydes, ketonic acids and alcohols. ${ }^{13,17}$ 4-hydroxybenzoic acid is a breakdown product of lignin. Dehydroabietic acid, vanillin and vanillic acids are specific compounds of conifers. ${ }^{6,14}$ The presence of methoxyphenols from lignin and resin acids indicates incineration of biomass. ${ }^{17}$

Methoxyphenols are the organic tracers for wood combustion. Direct photolysis converts vannilin or syringaldehyde into less volatile hydroxylated product such as vanillic or syringic acid. Syringaldehyde is a hardwood tracer compound, also present as minor amounts in softwood emissions. ${ }^{21,22}$

\section{7. Phthalate Esters}

The sample extracts contained a series of the polymeric materials' additives. Di(ethyl)phthalate (DEP), di(butyl)phthalate (DBP), di(2-ethylhexyl)phthalate (DEHP), di(isononyl)phthalate (DiNP), and di(isodecyl)phthalate (DiDP) are dominating compounds. The last two are present as a mixture of several isomers. Phthalate esters have a characteristic mass fragment $\mathrm{m} / \mathrm{z}$ 149. DEHP, DiNP and DiDP are mainly used as additives for softening PVC plastic. Evaporative releases from plastic material suggest the source for higher concentrations of phthalates during summer. Phthalates are possible carcinogens and endocrine disruptor compounds. ${ }^{19}$

\section{8. Secondary Organic Aerosol (SOA) Products of Terpenes}

Terrestrial vegetation emits large amounts of biogenic volatile organic compounds (BVOCs) into the atmosphere. Biogenic secondary aerosols (BSOA) are a fraction of secondary organic aerosols where the constituents are emitted from biogenic sources such as vascular plant waxes, micro-organisms, pollen, isoprene, monoterpenes etc. ${ }^{6}$ Figure 10 shows tracer compounds formed by the oxidation of cyclic monoterpene compounds. Pinonic acid and nopinone are oxidation products of monoterpenes $\alpha$-pinene and $\beta$-pinene. Terpenes are large and diverse class of organic compounds, produced by a variety of plants, particularly conifers. Pohorje Mountain is highly forested with conifer trees and is a large source of terpene compounds. Sabina ketone indicates transformation of monoterpene sa-

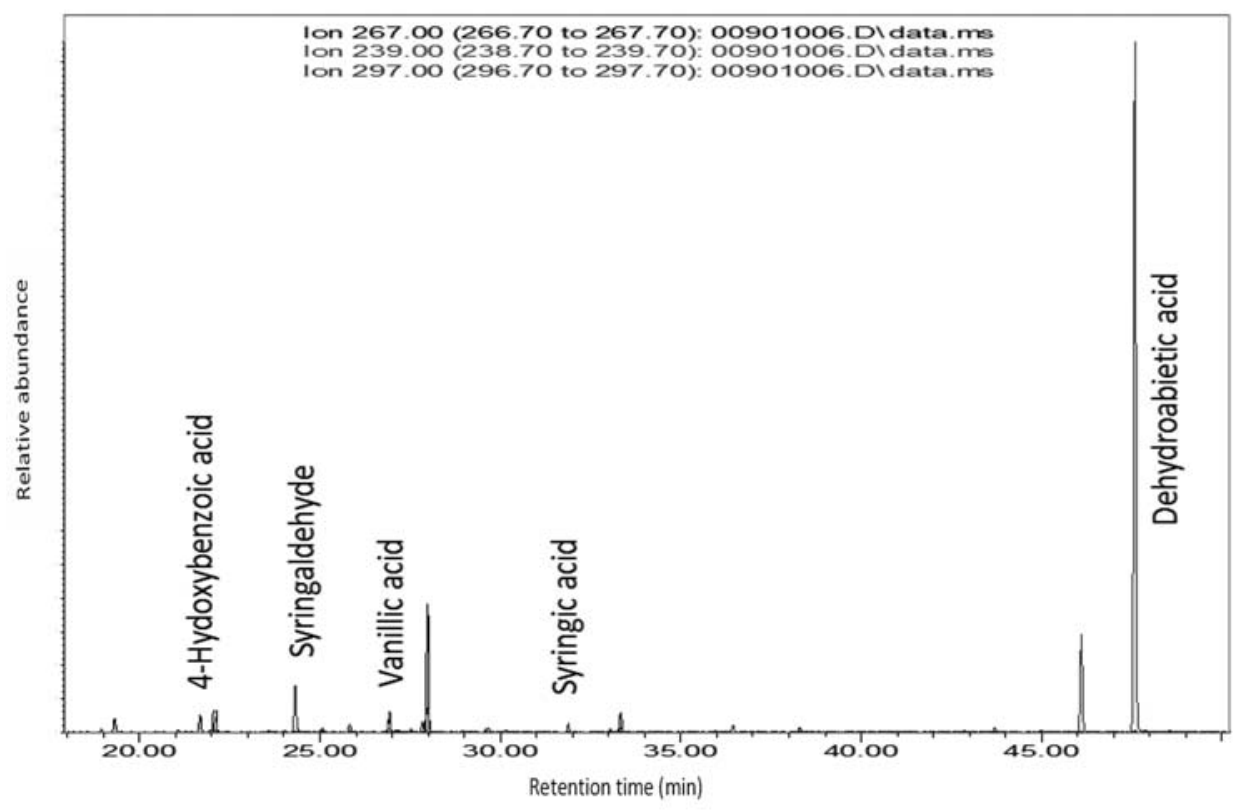

Figure 8. Selected ion chromatogram of lignin and resin acids from sample extracts (Collected: 12.5. 2014, MB VP). 


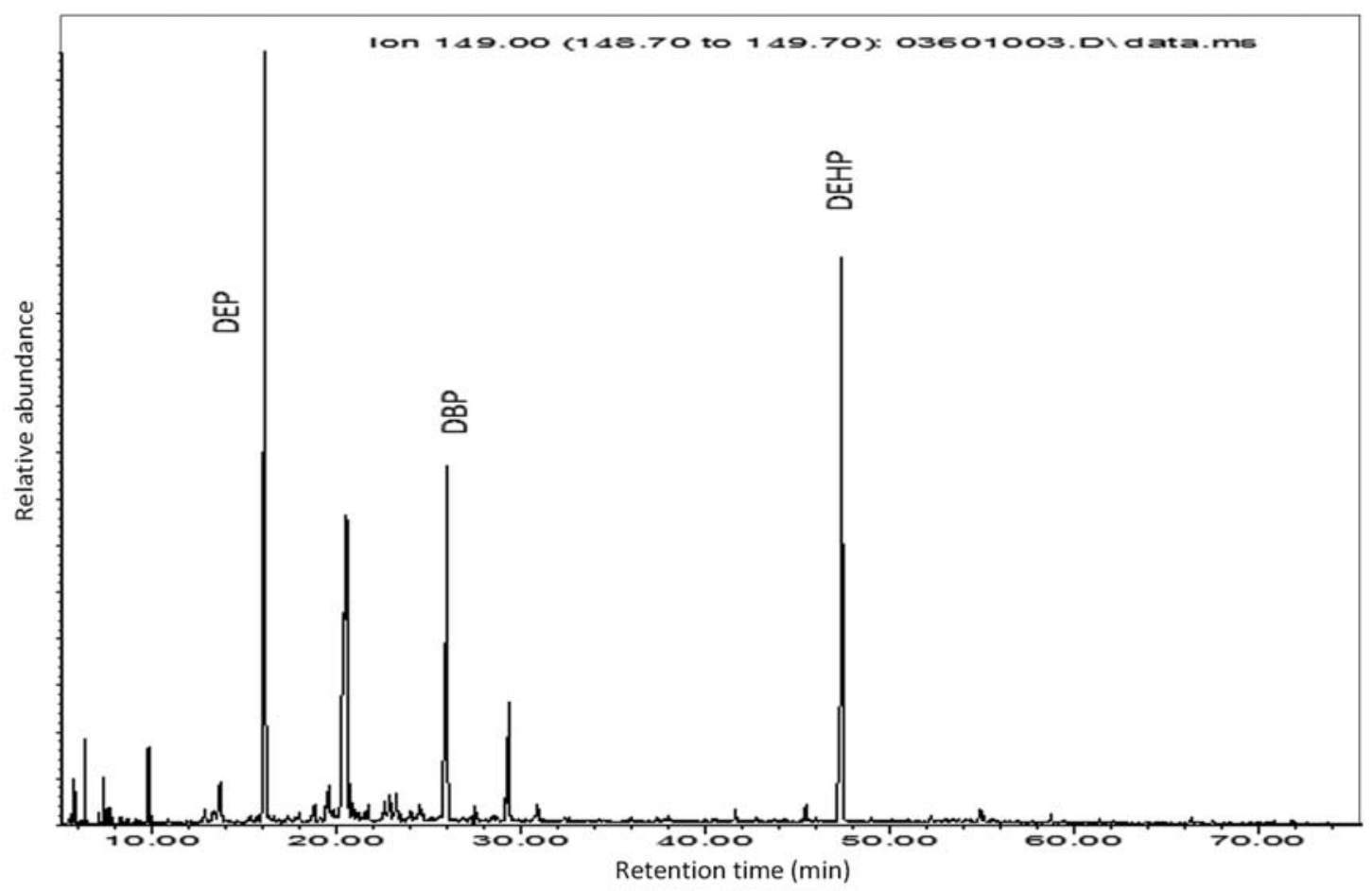

Figure 9. Chromatogram of phthalate esters from sample extracts (Collected: 18.7. 2014, MB NLZOH).

binene. Pinonaldehyde is the main product of the reaction between $\alpha$-pinene, ozone and $\mathrm{OH}$ radicals. ${ }^{6,17,23}$

In the sample extracts we detected 2-methyltetrols (2-methylthreitol and 2-methylerythritol) the oxidation products of isoprene. ${ }^{17}$ Photo-oxidation of isoprene is an important atmospheric chemical process that plays a unique role in the process of particles nucleation, contributes to the formation of SOA and causes »blue hazes« over forests. ${ }^{16,17,23}$

\section{9. Unresloved Complex Mixtures}

In several samples we detected unresolved complex mixtures (UCMs). UCMs appear in the chromatogram as large humps of complex mixture containing many components which are difficult to separate due to the large number of co-eluting compounds. ${ }^{23,24}$

It is evident from Figure 11 that the position of UCM in the chromatograms of silylated sample extract is different regarding the seasonal time. During the late

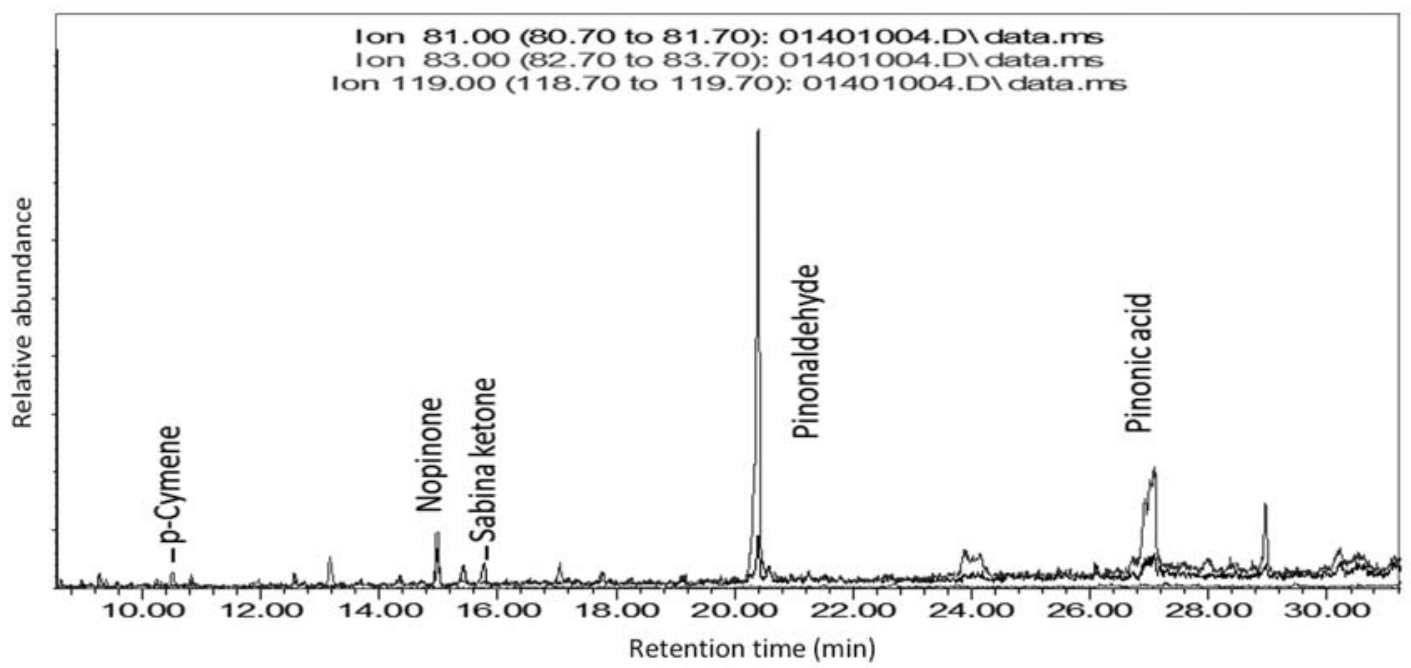

Figure 10. Ion current of selected mass fragments $\mathrm{m} / \mathrm{z} 81,83,119$ of monoterpenes photochemical oxidation products from sample extracts (Collected: 22.8. 2013, Miklavž). 


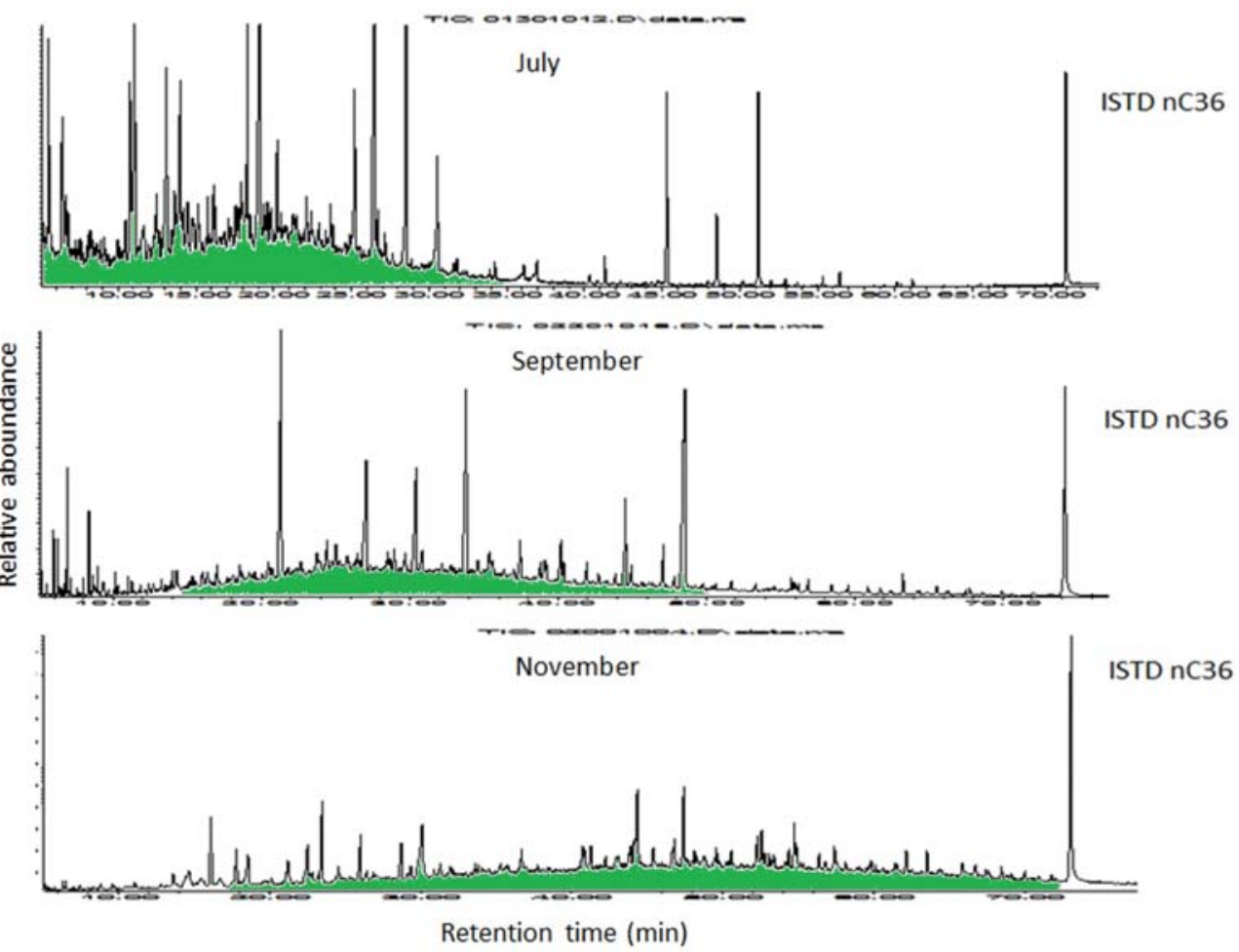

Figure 11. GC-MS chromatogram of detected UCMs from sample extracts in different seasons.

spring and summer atmospheric photo oxidation of VOCs may lead to the formation of particulate matter, also called SOA. These are polar organic compounds that are present in the air over the Maribor region. The production of biogenic precursors such as isoprene, pinene and other monoterpenes is increased in warmer seasons. This is due the influence of the Pohorje Mountain which is highly forested with coniferous trees. We used silylation, a derivatisation reaction to make polar compounds amenable for $\mathrm{GC}$ analysis. The formation of the silylated derivatives of SOA, namely polar polyfunctional compounds bearing labile hydrogens such as hydroxyl and carboxylic groups, provides analytes with lower polarities and better stability that enables the GC-MS identification.

During autumn and winter biogenic contribution to SOA is expected to be lower. Consequently classic UCM of branched and cyclic hydrocarbon compounds typical for fossil fuels and originating mostly from lubricating oils from vehicle engine exhausts predominate. ${ }^{24,25}$

\section{10. Siloxanes}

Producers of air samplers prescribe different adhesive coatings used for impaction surface such as Apiezon L. grease, silicone oil or silicone grease, polyisobutene, vaseline and others materials. ${ }^{32} 120$ samples were obtained using Silicon-Hochvakuumfett as it is recommended in standard EN12341:2014. 30 samples were additionally obtained using polyphenylether as a coating for the impact plate. Ae- rosols smaller or equal to $10 \mu \mathrm{m}$ pass through the sampler unaffected and are collected on the sampling filters. Aerosols greater than $10 \mu \mathrm{m}$ stroke and stuck on the greasy impact plate. Solid particles may be bounced from the surface of a greasy impact plate and re-entrained into the air stream and then collected on a sample filter. ${ }^{12,32}$ In such a way the certain part of silicones can be transferred on the sampling filter and can have an impact on the accuracy of PM10 gravimetric determination. Such "carryover" of siloxanes could disturb the gas chromatographic separation and determination of other organic compounds. In order to prevent contamination with siloxanes we experimentally used an adhesive material for the impact plate, polyphenylether (Santovac 5, diffusion pump oil is a 5-ring polyphenyl ether), with the adequate viscosity and sticky properties. This compound doesn't interfere with the chromatographic separation of other organic compounds, because it gives only one chromatographic peak eluting at the end of the chromatogram. We haven't detected siloxanes in sample extracts while using polyphenylether as a coating. Content of siloxanes in sample filter extracts was chromatographically quantitatively determined. The carryover of siloxanes was at least from $5 \%$ up to $15 \%$ of the accumulated particle's weight, depending on ambient temperature (from $20{ }^{\circ} \mathrm{C}$ to $30{ }^{\circ} \mathrm{C}$ during summer). This contribution of siloxanes in $50 \mathrm{~m}^{3}$ of sampled ambient air with the daily limit value of $50 \mu \mathrm{g} / \mathrm{m}^{3}$ for PM10 is the reason that gravimetric results for determination of PM10 sampled and measured according to the standard EN 12341:2014 were overestimated. 

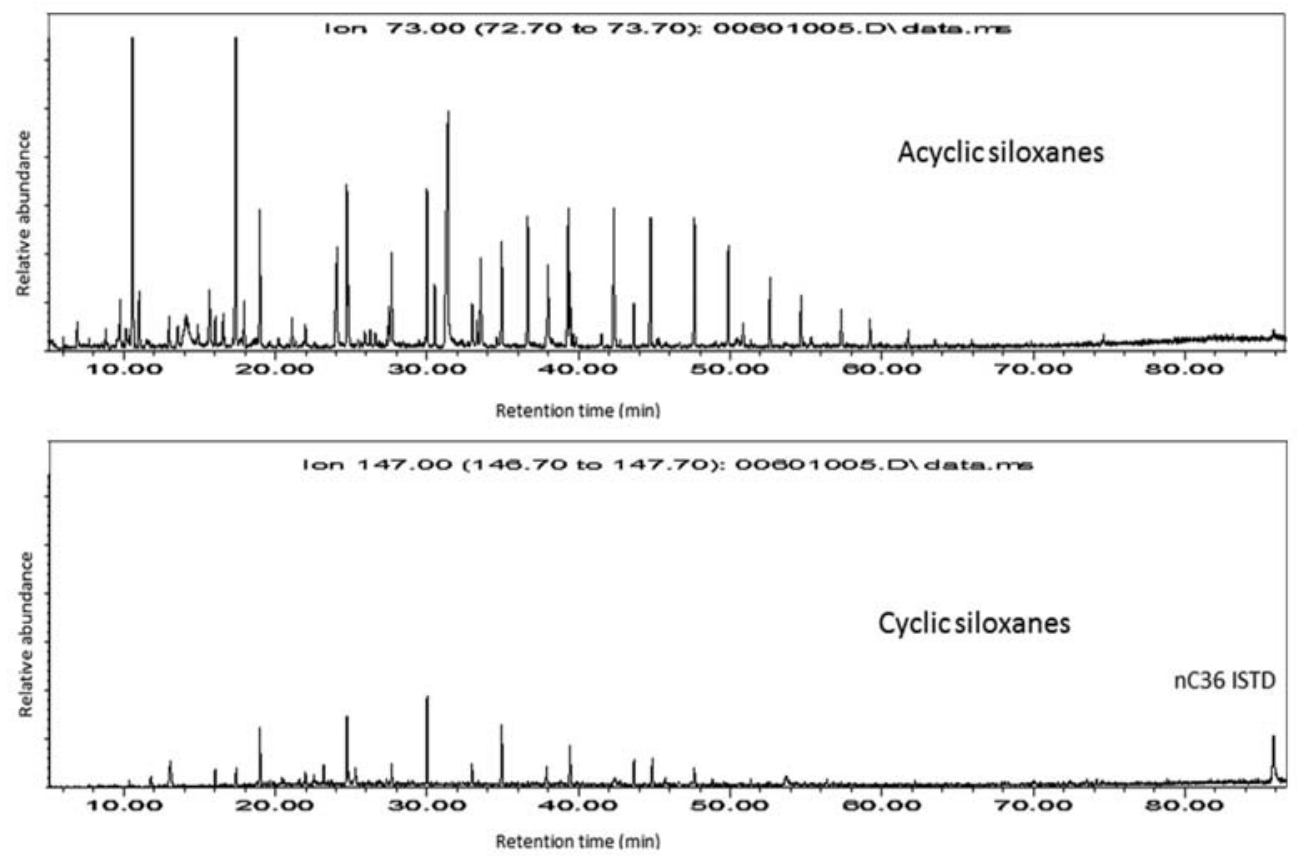

Figure 12. The chromatogram shows the distribution of anthropogenic cyclic and acyclic siloxanes from sample extracts (Collected: 22.7. 2013, MB VP).

Sample inlets should be cleaned and impact plates cleaned and greased according to the manufacturers requirements. ${ }^{12}$ We believe that it would be better to choose only one adhesive material with unique physicochemical properties. In such a way the influence of adhesive material on gravimetric PM10 determination could be better controlled.

\section{11. PAHs}

From the detected compounds and from the chromatographic data of samples gathered from Maribor district, it was evident that the presence of PAHs in comparison to other compounds was low during spring and summer but had increased concentrations during the heating season in

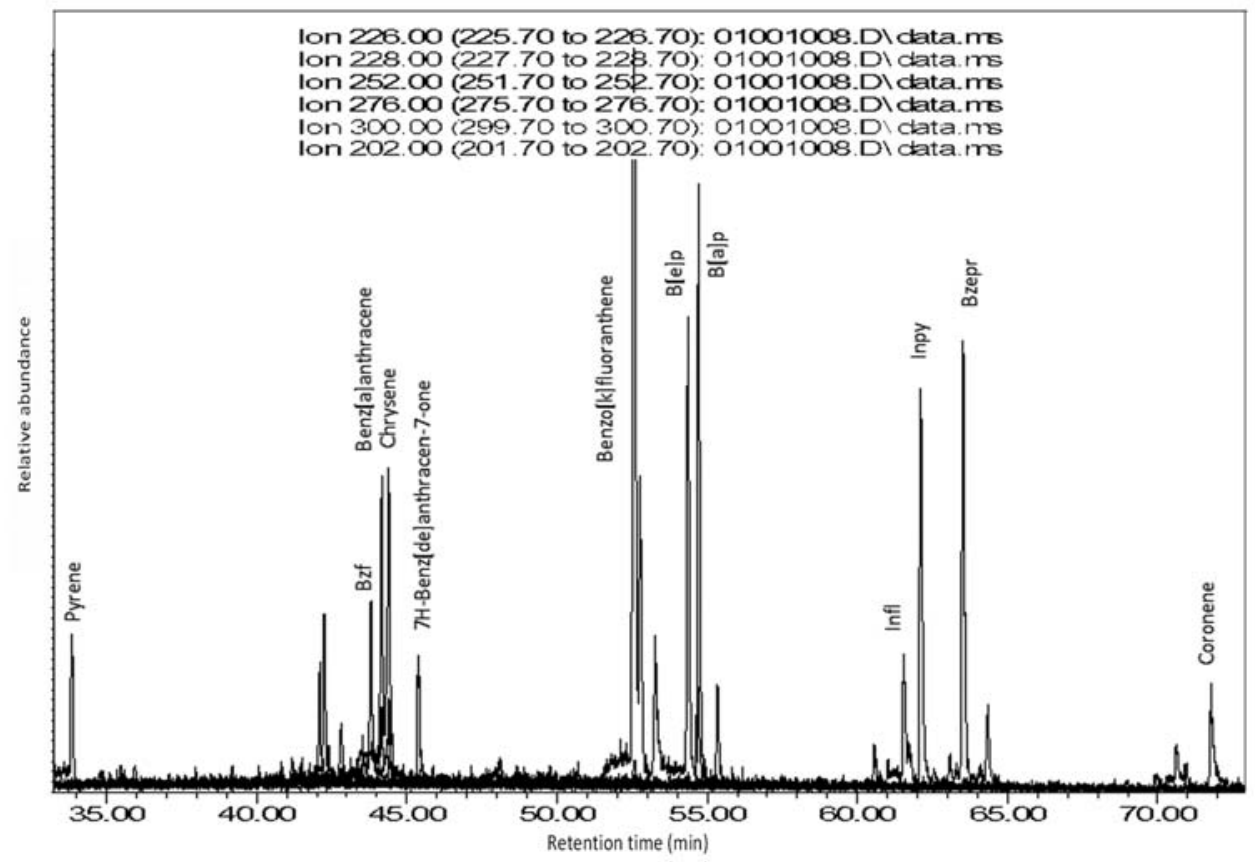

Figure 13. Chromatogram of PAHs distribution with selected ions $m / z$ 202, 226, 228, 252, 276, 300 from sample extracts (Collected: 19.12. 2013, MB NLZOH). 
autumn and winter with highest concentrations in December and January. Benzo[k]fluoranthene was the most abundant PAH on annual basis in the PM10 sample extracts of the city of Maribor.

PAHs are carcinogenic and mutagenic compounds naturally occurring in fossil fuels, their derived products can be formed during incomplete combustion of carbon based fuels. ${ }^{13,26}$ Combustion of fossil fuels is the main source of PAHs in the urban atmosphere. Domestic heating, industrial processes, energy production and natural sources (biomass burning, fire and vulcanic activity) are also responsible for the release of PAHs into the atmosphere. $^{17}$

\section{12. Other Detected Compounds}

Sitosterol is an indicator for vegetation in general. ${ }^{16}$ Cholesterol is emitted from meat cooking, fecal pollution and biological treatment plants. Nicotine is a tracer com- pound for tobacco smoke. ${ }^{17}$ Urea is a nitrogen-containing organic compound that might be directly emitted from active agricultural activities, biological waste water treatment plants or is a possible product of secondary photooxidation. ${ }^{6}$ The ketone 6,10,14-trimethylpentadecan-2one is a SOA and can be produced by thermal alteration and oxidation of phytol emitted from plants. ${ }^{17}$ Determination of organic compounds composition can help us to determine different local sources of air pollution. During industrial incidents air pollution in form of smoke, dust, smog or bad odour can appear. In that case air should be monitored within urban areas and in micro-environments relatively close to the emission's sources. Also the analysis of organic compounds in air should be performed. The method is suitable for the detection and tracking of local sources of pollution. In Figure 14 pollution from local industry in the city of Maribor is demonstrated, where lower molecular mass of polyoxymethylene (POM) has been detected.

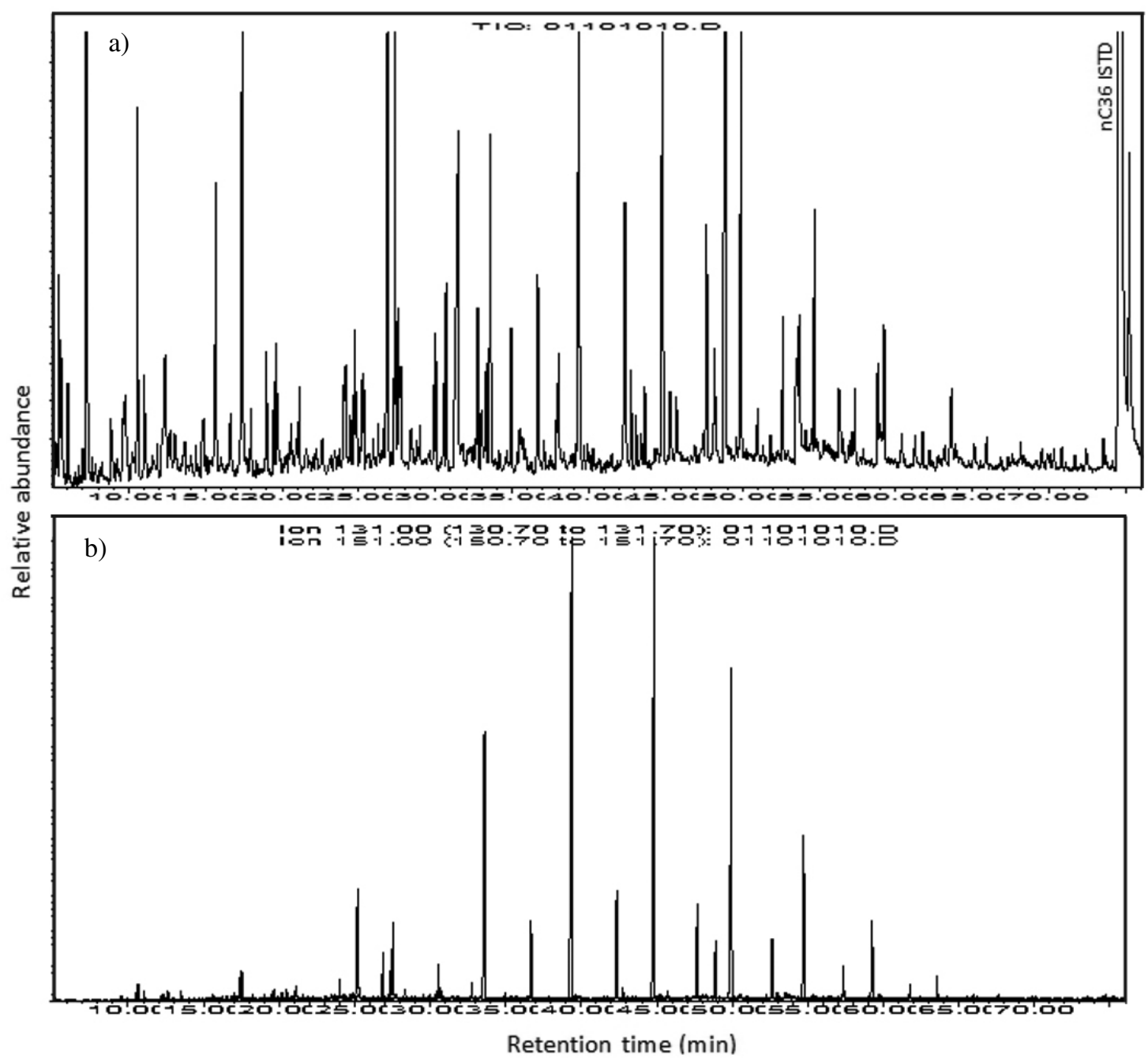

Figure 14. Chromatogram of sample PM10 extracts from an industrial zone in Maribor where impact of local industry is observed (A), characteristic selected mass fragments $\mathrm{m} / \mathrm{z} 131$ and $\mathrm{m} / \mathrm{z}, 151$ for polyol distribution $(B)$. 


\section{Conclusions}

Using the GC-MS analytical approach the composition of organic compounds in aerosols was determined. The suspended fraction of particulate matter PM10 in the air samples was analysed according to the standard EN 12341:2014 reference method. The research included 120 samples sampled during the period from summer 2013 to summer 2014. Beside gravimetric determination of PM10, the composition of organic compounds for estimation of pollution source was performed. During determination of organic compounds composition, the primary and secondary sources of air pollution were investigated. The technique of recording the full mass spectra has allowed us to monitor the presence of many pollution tracer compounds. The detected compounds included distributions of fatty acids, $n$-alkanes and iso-alkanes, phthalate esters, siloxanes, sterols, sugars and sugar alcohols, lignin tracer compounds and resin acids, dicarboxylic acids, polycyclic organic compounds, organic nitrogen compounds and products from secondary oxidation of monoterpenes. For the determination of volatile and semi-volatile polar organic compounds, the silylation was a prerequisite for good chromatographic analysis.

It was proven that the use of silicone grease for the purpose of lubricating the impact plate of the air sampler may cause higher values of gravimetric results. Quantitative determination was performed for siloxanes, which are a source for »carry over « and consequently are the reason for overestimated gravimetric results of PM10 determination according to the EN 12341:2014 reference method.

We demonstrated that the method is suitable for detecting and tracking air pollution from local sources. The research provides us with a better insight into composition of organic compounds in the atmosphere above Maribor.

\section{Acknowledgements}

The authors thank to the Slovenian Research Agency and the Ministry of Higher Education, Science and Technology of the Republic of Slovenia, contract number P1-017 and 41/2013-SOF for financial support and appreciate the technical assistance of Nino Javernik, from the National Laboratory of Health, Environment and Food, Maribor.

\section{References}

1. Official Journal of the European Union, Directive 2008/50/EC of the European Parliament and of the Council, http://eur-lex.europa.eu/legal-content/EN/TXT /PDF/?uri= CELEX:32008L0050\&from=en, (assessed: January 5, 2015)

2. Slovenian Environmental Agency (ARSO), Povprečne dnevne koncentracije delcev PM10 v januarju 2014, http://www. arso., 1gov.si/zrak/kakovost\%20zraka/podatki/PM10_D_dec2014_slo.pdf, (assessed: February 15, 2015)

3. J. D. Allan et al., Atmos. Chem. Phys, 2010, 10, 647-668. http://dx.doi.org/10.5194/acp-10-647-2010

4. T. Bolte et al., Opredelitev virov delcev PM10 v Sloveniji, http://www.arso.gov.si/zrak/kakovost\%20zraka/poro\%C4\%8Dila\%20in\%20publikacije/poro\%C4\%8Dila\%20o\%20projektih/pilotni_PM10.pdf, (assessed: August 28, 2014)

5. P. Q. Fu, K. Kawamura, Y. F. Cheng, S. Hatakeyama, A. Takami, H. Li, W. Wang, Atmospheric Chemistry and Physics, 2014, 14, 4185-4199. http://dx.doi.org/10.5194/acp-14-4185-2014

6. Official Journal of the European Union, Directive 2001/81/ EC of the European Parliament and of the Council, Official Journal, 2001, 309, 22-30.

7. L. Müller, M.-C. Reinning, K. H. Naumann, H Staathoff, T. F. Mentel, N. M. Donahue, T. Hoffmann, Atmos. Chem. Phys., 2012, 12, 1483-1496. http://dx.doi.org/10.5194/acp-12-1483-2012

8. P. Otorepec, K. Bitenc, I. Jeraj, N. Kovač, Zrak in zdravje, http://www.arso.gov.si\%2Fnovice\%2Fdatoteke\%2F031477Zrak\%2520MO\%2520Kranj\%2520februar\%25202014.docx \&ei=rYrjVPv9BofrUtCkgiA\&usg=AFQjCNHETXyGJdDF 3A1IpVURbmnoIWrriQ\&bvm=bv.85970519,d.bGQ, (assessed: December 13, 2014)

9. M. Hyder, J. Genberg, M. Sandahl, E. Swietlicki, J. Jönsson, Atmospheric Environment, 2012, 57, 197-204. http://dx.doi.org/10.1016/j.atmosenv.2012.04.027

10. Wikipedia The Free Encyclopedia, Smog, http://en.wikipedia.org/wiki/Smog, (assessed: August 28, 2014)

11._European Committee for Standardization, EN 12341:2014, Air quality - Determination of the PM10 fraction of suspended particulate matter, 2014.

12. B. R. T. Simoneit, M. Kobayashi, M. Mochida, K. Kawamura, M. Lee, H. J. Lim, B. J. Turpin, Y. Komazaki, Journal of Geophysical Research, 2004, 109, D19S10.

13. M. Xie, G. Wang, S. Hu, S. Gao, Q. Han, Y. Xu, J. Feng, Science of the Total Environment, 2010, 408, 5452-5460. http://dx.doi.org/10.1016/j.scitotenv.2010.07.029

14. A. Limbeck, Y. Kraxner, H. Puxbaum, Aerosol Science, 2005, 36, 991-1005. http://dx.doi.org/10.1016/j.jaerosci.2004.11.013

15. K. Kawamura, H. Kasukabe, L. Barrie, Atmospheric Environment, 1996, 30, 1709-1722. http://dx.doi.org/10.1016/1352-2310(95)00395-9

16. C. A. Alves, Anais da Academia Brasileira de Ciências, 2008, 80(1), 21-82. http://dx.doi.org/10.1590/S0001-37652008000100003

17. Y. Cheng, S. M. Li, A. Leithead, J. R. Brook, Atmospheric Environment, 2006, 40, 2706-2720.

http://dx.doi.org/10.1016/j.atmosenv.2005.11.066

18. T. Gnauk, A. Plweka, , O. Böge, E. Brügegemann, , D. Hofmann, Y. Iinuma, H. Herrmann, Particle modification and formation from BVOC emissions above Coniferous Forest in Germany, 
http://imk-ifu.fzk.de/bewa2000/openMaterial/ BEWAPoster_Gnauk.pdf, (assessed: December 11, 2014)

19. W. Kirch, A. Müller-Schuchardt, A. Zscheppang, I Want To Know What I Am Breathing,

http://www.ufireg-central.eu/files/Downloads/UFIREGBroschuere_de.pdf, (assesed: January 25, 2015)

20. M. A. Bari, G. Baumbach, B. Kuch, G.Scheffknecht, Aerosol and Air Quality Research, 2011, 11, 749- 757.

21. Y. J. Li, D. D. Huang, H. Y. Cheung, A. K. Y. Lee, C. K. Chan, Atmos. Chem. Phys., 2014, 14, 2871-2885. http://dx.doi.org/10.5194/acp-14-2871-2014

22. I. Kourtchev, T. Ruuskanen, W. Maenhaut, M. Kulmala, M. Claeys, Atmos. Chem. Phys., 2005, 5, 2761-2770. http://dx.doi.org/10.5194/acp-5-2761-2005

23. G. S. Frysinger, R. B. Gaines, L. Xu, C. M. Reddy, Environ. Sci. Technol., 2003, 37(8), 1653-1662. http://dx.doi.org/10.1021/es020742n

24. G. T. Ventura, F. Kenig, C. M. Reddy, G. S. Frysinger, R. K. Nelson, B. V. Mooy, R. B. Gaines, Organic Geochemistry, 2008, 39, 846-867. http://dx.doi.org/10.1016/j.orggeochem.2008.03.006

25. N. Falquet, G. d'Esperonnat, R. Darrington, Evaluation of an Improved Sample Preparation Method for Quantative Analysis of Very Low Levels of Airborne Polycyclic Aromatic Hydrcarbons for Worker Protection and Health Screening, http://www.labrepco.com/data/file-downloads/Improved_Yield_During_Safe_Sample_Concentration_ 1371492467.pdf, (assessed: November 8, 2014)
26. G. Wang, K. Kawamura, T. Watanabe, S. Lee, K. Ho, J. Cao, Geophysical Research Letters, 2007, 33, L22801.

27. T. Gnauk, A. Plweka, , O. Böge, E. Brügegemann, , D. Hofmann, Y. Iinuma, H. Herrmann, Particle modification and formation from BVOC emissions above Coniferous Forest in Germany,

http://imk-ifu.fzk.de/bewa2000/openMaterial/ BEWAPoster_Gnauk.pdf, (assessed: December 11, 2014)

28. C. M. Reddy, T. I. Eglinton, R. Palić, B. Nelson, G. Stojanović, I. Palić, S. Djordjević, G. Eglinton, Organic Geochemistry, 2000, 31, 331-336. http://dx.doi.org/10.1016/S0146-6380(00)00025-5

29. M. Glasius, M. Lahaniati, A. Calogirou, D. Di Bella, N. R. Jensen, J. Hjorth, D. Kotzias, B. R. Larsen, Environ. Sci. Technol., 2000, 34, 1001-1010. http://dx.doi.org/10.1021/es990445r

30. M. S. Alam, C. E. West, A. G. Scarlett, S. J. Rowland, R. M. Harrison, Atmospheric Environment, 2013, 65, 101-111. http://dx.doi.org/10.1016/j.atmosenv.2012.10.014

31. N. E. Sanchez, J. Salafranca, A. Callejas, A. Millera, R. Bilbao, M. U. Alzueta, Fuel, 2013, 107, 246-253. http://dx.doi.org/10.1016/j.fuel.2013.01.065

32. S. V. Hering, Impactors, Cyclones, and Other Inertial and Gravitational Collectors, http://158.110.32.35/CLASS/DES-IND-PLA1/impactorscyclonesch14p1.pdf, (assessed: February 22, 2015)

\section{Povzetek}

V 120 vzorcih PM10 smo analizirali organske spojine v aerosolskih delcih v zraku nad Mariborom z uporabo metode EN 12341:2014. Proučevali smo sestavo organskih spojin ter primarne in sekundarne izvore le-teh. Za določevanje hlapnih in delno hlapnih polarnih organskih spojin z GC-MS smo uporabili sililiranje kot derivatizacijsko metodo. Določevali smo porazdelitve maščobnih kislin, $n$-alkanov in izo-alkanov, ftalatnih estrov, siloksanov, različnih sterolov, različnih sladkorjev in sladkornih alkoholov, spojine ligninov in smolnih kislin, dikarboksilnih kislin nastalih s fotokemijskimi reakcijami, policikličnih organskih ogljikovodikov, organskih dušikovih spojin in produktov nastalih pri sekundarni oksidaciji monoterpenov. Uporaba silikonske masti na površini impaktne plošče vzorčevalnika lahko povzroči višje vrednosti gravimetrijske določitve. Trdni delci se lahko odbijejo od impaktne plošče in ponovno vstopijo v zračni tok in se nato zberejo na filtru. To prenašanje siloksanov je lahko najmanj od 5\% do $15 \%$ skupne mase akumuliranih delcev v odvisnosti od temperature. To je razlog, da so gravimetrijski rezultati določevanja PM10 po EN 12341:2014 standardu previsoki. 